Proceeding Paper

\title{
Dispensing of Food Supplements in the Treatment and Prevention of Urinary Tract Infections ${ }^{\dagger}$
}

\author{
Beatriz Setoca and Ana I. Fernandes *(i)
}

check for

updates

Citation: Setoca, B.; Fernandes, A.I. Dispensing of Food Supplements in the Treatment and Prevention of Urinary Tract Infections. Med. Sci. Forum 2021, 5, 1. https://doi.org/ $10.3390 / \mathrm{msf} 2021005001$

Academic Editors: Helena Barroso and Cidália Castro

Published: 9 July 2021

Publisher's Note: MDPI stays neutral with regard to jurisdictional claims in published maps and institutional affiliations.

Copyright: (c) 2021 by the authors. Licensee MDPI, Basel, Switzerland. This article is an open access article distributed under the terms and conditions of the Creative Commons Attribution (CC BY) license (https:// creativecommons.org/licenses/by/ $4.0 /)$.
CiiEM, Interdisciplinary Research Center Egas Moniz, Instituto Universitário Egas Moniz, Quinta da Granja, Monte de Caparica, 2829-511 Caparica, Portugal; beatrizdfpsetoca@gmail.com

* Correspondence: aifernandes@egasmoniz.edu.pt; Tel.: +351-212946823

+ Presented at the 5th International Congress of CiiEM-Reducing Inequalities in Health and Society, Online, 16-18 June 2021.

Abstract: Urinary tract infections constitute an important public health issue due to recurrence and antibiotic resistance. Currently, antibiotics are the standard therapy but non-antibiotic approaches, such as food supplements, could be beneficial and reduce bacterial resistance. This work aimed at a better understanding of the perception of health professionals involved in dispensing and counselling, in the community pharmacy, regarding the utility of these products as preventive alternatives and therapeutic approaches.

Keywords: food supplements; urinary tract infections; prevention; treatment

\section{Introduction}

Urinary tract infections (UTI) are one of the most common infections, both in healthcare and in the community setting [1]. Currently, antibiotics (e.g., fosfomycin) are the standard therapy, but the high rates of infection recurrence and antibiotic resistance pose an important public health problem. With that in mind, the management of this pathology using non-antibiotic therapies, such as food supplements (FS), are considered an asset and could reduce resistance. However, the use of FS in the treatment and prevention of UTI is still controversial, since the associated risks and benefits are unknown to many health professionals (HP) [2].

An in-depth study of the scientific evidence available is; therefore, warranted to enable informed dispensing and counselling of these products in community pharmacies. The aim of this study was to evaluate the consumption of FS for the prophylaxis and/or treatment of UTI in Portugal and assess differences of pharmacist's behavior vs non-pharmacists towards their recommendation.

\section{Materials and Methods}

Sales data (2017 to 2019) of FS indicated in UTI management were obtained from the National Pharmacies Association (ANF), representing about $95 \%$ of the national pharmacies. Eighty-five FS were analyzed to identify the alleged bioactive molecules and the labelling of the five top sellers examined, from the perspective of efficacy and safety. To characterize the dispensing and counselling act, in the context of treatment/prevention of UTI, an anonymous online questionnaire (approved by Egas Moniz's Ethics Committee) was applied to HP working in a community pharmacy (inclusion criteria). Besides pattern of dispensing/counselling, preference and the perception of quality, safety and efficacy were also evaluated. The questionnaire was shared on social media, in specific professional groups, and emailed to 200 community pharmacies. Responses $(n=149)$ were collected between April and October 2020. The study was complemented with a literature review in databases, such as Cochrane Library and PubMed. 


\section{Results and Discussion}

Results show increased ( 44\%) dispensing of FS for UTI, in pharmacies, from 2017 to 2019, which may suggest either an increasing prevalence of UTI or a higher demand of these products by consumers. The top selling FS are sold as hard gelatin capsules and present a great diversity of alleged bioactives (mainly Vaccinium macrocarpon-cranberry, Arctostaphylos uva-ursi-bearberry and Lactobacillus sp.-probiotics), many of them combined in the same formulation, which potentiates adverse reactions and interactions. Required legal labelling mentions were present in every product; indication of the maximum/ideal duration of consumption and minimum age for intake were lacking. Many products recommended that a HP should be notified in cases of combined use of anticoagulants, antiplatelet agents and in pregnancy, or lactation. Yet, since there are no official recommendations, it is up to the HP to advise, or not, the use of FS in these situation.

Most of the respondents to the questionnaire $(n=149)$, involved in FS dispensing and counselling, were pharmacists $(78 \%)$; technicians and assistant technicians account for the remaining professionals. FS for UTI are dispensed frequently (once/twice a week) in the pharmacy, by $42 \%$ of the professionals; $92 \%$ of these dispensing acts are reported as accompanied by counselling.

Although FS cannot claim the treatment or prevention of disease, a majority of the HP believe that FS are important in UTI, as adjuvants to drug therapy $(n=122)$, in prevention $(n=93)$ or even as treatment $(n=59)$. Although clinical studies and meta-analysis available in literature do not clearly demonstrate the efficacy of the FS in UTI [2], only a minority of the HP $(n=4)$ believe that they are not important in these infections, due to lack of safety $(0.7 \%)$ or inefficacy $(2 \%)$.

An independent question cross-checked perception of quality, efficacy and safety with respect to FS. Again, only $12 \%$ of the professionals consider that FS do not attain the minimum requisites; the vast majority of pharmacists consider FS as being safe and of quality. Efficacy is perceived only by a minority (13\%) of non-pharmacists; perception of pharmacists is in line with the existing lack of evidence regarding these products, found in the literature. HP in community pharmacies, represented by $91 \%$ of pharmacists, $93 \%$ of technicians and $83 \%$ of assistant technicians, believe that regulation and inspection of FS should be similar to that of medicines. A significant number of pharmacists also consider that publicity $(16 \%)$ and regulation $(11 \%)$ of FS should be reviewed, though retaining the status of foods. The need to include FS in the pharmacovigilance system, providing monitoring regarding potential interactions and contraindications, was also mentioned.

The majority of HP (54\%) reported not to indicate a specific product (brand) to the consumer. Pharmacists ( $47 \%$ ) are those who tend to have a product of election; choice is justified by perception of efficacy previously reported, or confidence in the manufacturer of the FS. The main bioactives indicated were cranberry $(59 \%)$ and bearberry $(14 \%)$, coinciding with the consumption data.

In conclusion, though HP in community pharmacies consider FS as a safe and important non-pharmacological approach in the management of UTI, there is agreement with the need for further scientific evidence on safety and efficacy, as well as to bring FS regulation closer to that of medicines.

Funding: This research received no external funding.

Institutional Review Board Statement: The study was conducted according to the guidelines of the Declaration of Helsinki, and approved by the Ethics Committee of Egas Moniz (protocol code 799; date of approval: 19 December 2019).

Informed Consent Statement: Informed consent was obtained from all subjects involved in the study.

Acknowledgments: ANF is acknowledged for providing the FS sales data.

Conflicts of Interest: The authors declare no conflict of interest. 


\section{References}

1. Medina, M.; Castillo-Pino, E. An introduction to the epidemiology and burden of urinary tract infections. Ther. Adv. Urol. 2019, 11. [CrossRef] [PubMed]

2. Cai, T.; Tamanini, I.; Kulchavenya, E.; Perepanova, T.; Köves, B.; Wagenlehner, F.M.; Tandogdu, Z.; Bonkat, G.; Bartoletti, R.; Johansen, T.E.B. The role of nutraceuticals and phytotherapy in the management of urinary tract infections: What we need to know? Arch. Ital. Urol. Androl. 2017, 89, 1-6. [CrossRef] [PubMed] 